domain translocates to the nucleus. Tschopp and colleagues then showed that supernatants that contain recombinant Helicard sensitized non-apoptotic extracts to DNA fragmentation.

Although Helicard has no intrinsic DNase activity, the authors speculate that it might facilitate CAD-mediated cleavage. It probably does this through its helicase activity, which opens up the DNA to allow easier access for CAD - a clever twist indeed. Alison Mitchel

6) References and links ORIGINAL RESEARCH PAPER Kovacsovics, M. et al. Overexpression of Helicard, a CARDcontaining helicase cleaved during apoptosis, accelerates DNA degradation. Curr. Biol. 12 838-843 (2002)

its inactivation. It will be interesting to establish how DIAP1-mediated ubiquitylation of Dronc leads to its inactivation, as the authors have preliminary evidence is that it does not occur by proteasome degradation.

These findings put RHG protein signalling into a new perspective, but they also raise many questions about the link between Morgue and E3 ligase complexes, and about the mechanism by which RHG proteins regulate translation.

Valerie Ferrier, Associate Editor, Nature Cell Biology

(0) References and links ORIGINAL RESEARCH PAPERS Yoo, S. J. et al. Hid, Rpr and Grim negatively regulate DIAP1 levels through distinct mechanisms. Nature Cell Biol.4. 416-424 (2002) | Hays, R., Wickline, L. \& Cagan, R. Morgue mediates apoptosis in the Drosophila melanogaster retina by promoting degradation of DIAP1. Nature Cell Biol. 4, 425-431 (2002) |Ryoo, H. D., Bergmann, A., Gonen, H., Ciechanover, A. \& Steller, H. Regulation of Drosophila IAP1 degradation and apoptosis by reaper and $u b c D 1$. Nature Cell Biol. 4, 432-438 (2002) | Holley, C. L. Olson, M. R., Colón-Ramos, D. A. \& Kornbluth, S. Reaper eliminates IAP proteins through stimulated IAP degradation and generalized translational inhibition. Nature Cell Biol. 4, 439-444 (2002)| Wilson, R. et al. The DIAP1 RING finger mediates ubiquitination of Dronc and is indispensable for regulating apoptosis. Nature Cell Biol. 4, 445-450 (2002) | Wing, J. P. et al. Drosophila Morgue is an F box/ubiquitin coniugase domain protein important for grim-reaper mediated apoptosis. Nature Cell Biol. 4, 451-456 (2002)

WEB SITES

Hermann Steller's laboratory:

http://www.rockefeller.edu/labheads/steller/ stellerhtml

Ross Cagan's laboratory:

http://molecool.wustl.edu/caganlab.htm Sally Kornbluth's laboratory:

http://cmb.duke.edu/faculty/kornbluth.html

Bruce Hay's laboratory:

http://www.its.caltech.edu/ biology/brochure/facu ty/hay.html

\section{Destination lipid rafts}

The cell is a mêlée of reactions, so dividing some of these reactions into specific subcompartments should help increase their specificity and efficiency. One such compartment is the plasma membrane, which is further subdivided into lipid rafts and caveolae - microdomains that are enriched in cholesterol, sphingolipids and signalling proteins. But how do proteins reach these destinations? According to a report in Science by Roger Tsien's group, acylation is sufficient to preferentially localize fluorescent test proteins to lipid rafts.

Their studies involved the fluorescence resonance energy transfer (FRET) technique using the cyan and yellow fluorescent protein (CFP and YFP, respectively) variants of green fluorescent protein (GFP). As these proteins lack endogenous targeting sequences, the aim was to study the effect of adding consensus sequences for acylation - the addition of myristoyl and/or palmitoyl moieties — or prenylation — the addition of farnesyl or geranylgeranyl moieties - on the localization of these proteins in membrane microdomains of live cells. Individual microdomains are so small that they are beyond the resolution of standard light microscopy, so the authors relied on the greater spatial resolution that is provided by FRET to determine which lipid-modified fluorescent proteins were selectively sequestered within them. The hallmark of this clustering is that there is an association - as measured by FRET - even when very little of the protein is expressed on the plasma membrane.

Initially, Tsien and colleagues used the acylation substrate sequence from the kinase Lyn to promote the attachment of myristoyl and palmitoyl chains to the amino termini of CFP and YFP. The FRET efficiencies were high, which indicates that the proteins were close to each other and therefore, they presumed, in the microdomains. But as treatment with 5-methyl$\beta$-cyclodextrin $(\mathrm{M} \beta \mathrm{CD})$ - a compound that depletes cholesterol and therefore disrupts lipid rafts and caveolae - did not reduce the interactions, they suspected that the fluorophores were themselves dimerizing, and giving a false impression of clustering. So, they changed the hydrophobic residues that are found at the dimerization interface of CFP and YFP to positively charged residues, which prevented dimer formation.

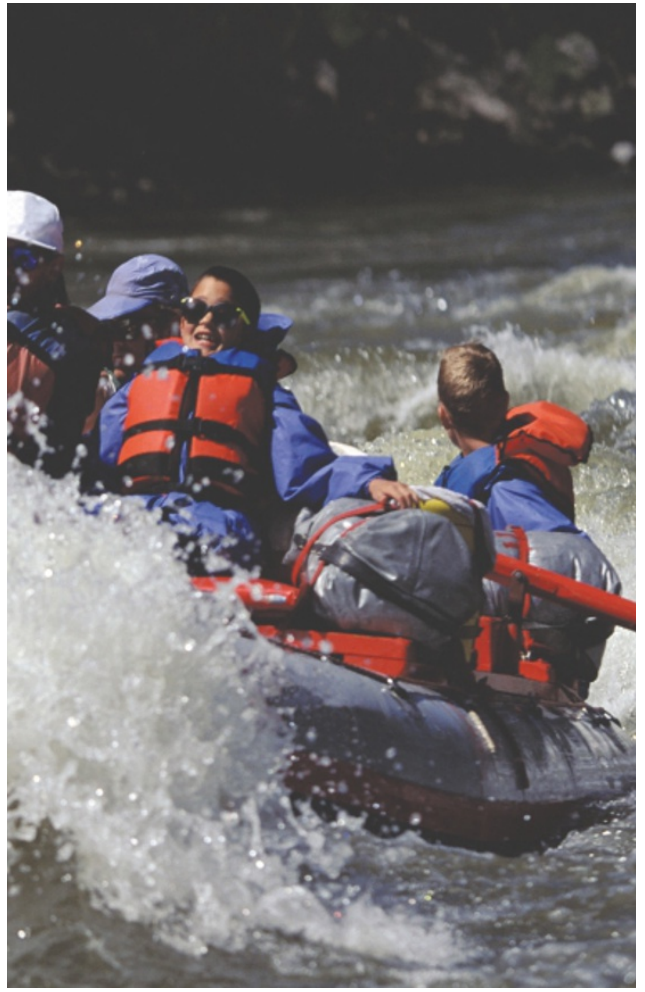

Adding the amino terminus of Lyn and expressing these 'monomeric' $(m)$ variants in cells resulted in increased FRET efficiency that, this time, was destroyed by $M \beta C D$, which indicates that the clustering can be attributed to the localization of these proteins in microdomains. Furthermore, the authors showed that acylation promoted the association of both of the monomeric variants with caveolin, a marker for caveolae.

By contrast, geranylgeranylated versions of mCFP or mYFP - studied by fusing the CAAX box of Rho to their carboxyl termini -

clustered with themselves, but not with acylated versions or with caveolin, which indicates that prenylation induces clustering, but not into the same membrane microdomain as the acylated fluorescent proteins or caveolae.

To confirm these findings, the authors reverted to traditional biochemical fractionation assays using the MYFP variant. Myristoylated and palmitoylated YFP separated mainly into detergent-soluble fractions with low-density caveolae-rich membranes - in the same manner as endogenous caveolin - which indicates that it had separated with caveolae. Geranylgeranylated YFP, on the other hand, was excluded from these fractions. Proof, then, that acylation, but not prenylation, will target you to a lipid raft!

Katrin Bussell

\section{6) References and links}

ORIGINAL RESEARCH PAPER Zacharias, D. A., Violin, J. D. Newton, A. C. \& Tsien, R. Y. Partitioning of lipid-modified monomeric GFPs into membrane microdomains of live cells. Science 296, 913-916 (2002)

FURTHER READING Simons, K. \& Toomre, D. Lipid rafts and signal transduction. Nature Rev. Mol. Cell Biol. 1, 31-39 (2000) Lippincott-Schwartz, J. et al. Studying protein dynamics in living cells. Nature Rev. Mol. Cell Biol. 2, 444-456 (2001) WEB SITES

Encyclopedia of Life Sciences: http://www.els.net Green fluorescent protein

Roger Tsien's laboratory: http://www.tsienlab.ucsd.edu 